\title{
Schrödinger Operators on Graphs and Branched Manifolds
}

\author{
M. H. Numan Elsheikh \\ Department of Differential Equations and Mathematical Physics, Peoples’ Friendship University of Russia, Moscow, Russia \\ Email:
}

Received December 3, 2013; revised January 3, 2014; accepted January 9, 2014

Copyright (c) 2014 M. H. Numan Elsheikh. This is an open access article distributed under the Creative Commons Attribution License, which permits unrestricted use, distribution, and reproduction in any medium, provided the original work is properly cited. In accordance of the Creative Commons Attribution License all Copyrights @ 2014 are reserved for SCIRP and the owner of the intellectual property M. H. Numan Elsheikh. All Copyright (C) 2014 are guarded by law and by SCIRP as a guardian.

\begin{abstract}
We consider the Schrödinger operators on graphs with a finite or countable number of edges and Schrödinger operators on branched manifolds of variable dimension. In particular, a description of self-adjoint extensions of symmetric Schrödinger operator, initially defined on a smooth function, whose support does not contain the branch points of the graph and branch points of the manifold. These results are obtained for graphs with a single vertex, graphs with multiple vertices and graphs with a single vertex and countable set of rays.
\end{abstract}

\section{KEYWORDS}

The Schrödinger Equation; Schrödinger Operators on Graphs and Branched Manifolds; Self-Adjoint Extensions

\section{Introduction}

Differential operators on graphs and other branched manifolds have applications to the description of a number of processes in quantum mechanics and biology. Fundamentals of the theory of differential equations on graphs presented in the monograph [1], in which a number of examples of physical problems leading to the study of differential operators on graphs. In the articles [2-4] spectral properties of such operators are investigated by the dynamic properties of the evolution determined by the Schrödinger equation on the graph. In the articles [5-8] we study the set of self-adjoint extensions Schrödinger operator defined initially in the space of compactly supported smooth functions, whose support do not contain the branch points of the graph ([6-8]) or points of changing the operator type [5]. Feynman approximation formulas for the unitary semigroups defined by some of the self-adjoint extensions are founded in the article [7]. This article contains the consideration of the Laplace operators on graphs with a finite or countable number of edges. This article is a continuation of studies [7] in which we studied the graph with a finite set of edges are considered.

The relevant problem under consideration consists of recently considerable interest in the description of particle dynamics on graphs, branched dendrites and other manifolds from mathematical physics and quantum mechanics. Mathematically, the operation of differentiation function is uniquely defined for functions on region or on a smooth manifold, which needs to be clarified for the functions defined on manifolds, containing the branch point. The purpose of this study is to determine the action of the Schrödinger operator on functions defined on a manifold with a finite set of branch points. For this purpose, we define the Schrödinger operator $\mathbf{L}_{0}$ in the space $C_{0,0}^{\infty}$ finite and infinitely differentiable functions whose support does not contain the branching points. Schrödinger operator $\mathbf{L}$ on a graph is called a self-adjoint extension of the operator $\mathbf{L}_{0}$. In this article we describe the set of all operators of Schrödinger operators on a graph in terms of conditions on the set of limit values at the branch point functions in the domain of $\mathbf{L}$ and its derivative. In this article we obtained the results with a single vertex (they represent a union $n$ of semidirect with a common vertex), graphs with multiple vertices and graphs with a single vertex and a countable set of rays and the set of all operators of Schrödinger on branched 
manifold in terms of conditions on the set of limit values on a manifold of branching functions in the domain of the operator $\mathbf{L}$.

In this article we found general description of a set of self-adjoint extensions, of the operator $\mathbf{L}_{0}$, as on graphs and branched manifolds.

\section{Formulation of Problem and Notation}

We study the Schrödinger operator on the graph $\Gamma$, defining the processes of diffusion and quantum dynamics on a graph both on branched manifold. Following [1] terminology graph $\Gamma$ is called finite or countable collection of smooth one-dimensional manifolds $\Gamma_{i}$ (called edges of the graph), each of which is diffeomorphic to the ray $[0,+\infty)$ or interval $[0,1]$. The boundary points of the edges will be called vertices of the graph. Each vertex of a graph is a boundary point of a non-empty set of edges of a graph.

Assumed that on $\Gamma$ given Borel measure, we determine the requirement that its restriction to each edge $\Gamma_{\mathrm{j}}$ coincides with the standard Lebesgue measure, then $L_{2}(\Gamma)=\oplus L_{2}\left(\Gamma_{j}\right)$.

Let $C_{0,0}^{\infty}(\Gamma)$-vector space of infinitely differentiable complex-valued functions on $\Gamma$ with compact support not containing the vertices, and operator $\mathbf{L}_{0}=\oplus \mathbf{L}_{0}^{j}$ is linear operator defined on a linear space $\mathrm{D}\left(\mathrm{L}_{0}\right)=\mathrm{C}_{0}^{\infty}(\Gamma)$ by the equation

$$
\mathbf{L}_{0} \mathrm{u}=\frac{1}{\mathrm{~m}} \Delta \mathrm{u}+\mathrm{iB}(\mathrm{x}) \frac{\partial \mathrm{u}}{\partial \mathrm{x}}+\mathrm{i} \frac{\partial(\mathrm{B}(\mathrm{x}) \mathrm{u})}{\partial \mathrm{x}}+\mathrm{C}(\mathrm{x}) \mathrm{u},
$$

in which the functions $\mathrm{m}, \mathrm{B}, \mathrm{C}$-real-valued, bounded and continuous everywhere except at the vertex function on $\Gamma$, function $\mathrm{m}$ takes on each edge $\Gamma_{j}$ a constant value $\mathrm{m}_{j}$, and $\mathrm{m}_{j} \geq \mathrm{m}_{0}>0$ for all $j=1, \cdots, n$; $\mathrm{u} \in C_{0}^{\infty}(\Gamma)$.

We say that $\Gamma$ is branched manifolds, if $\Gamma$ defined as the union of $n$ instances of regions $\Gamma_{\alpha}, \quad \alpha \in\{1,2, \cdots, n\}$ : $\Gamma=\bigcup_{\alpha=1}^{n} \Gamma_{\alpha}$, we assume that for each $\alpha$ region $\Gamma_{\alpha}$ is $d_{\alpha}$-dimensional bounded domain in the space $R^{d_{\alpha}}$ with $\left(d_{\alpha}-1\right)$ dimensional smooth boundary $\eta_{\alpha}$. The boundary of the manifold $\Gamma$ is defined as the union of $n$ instances of boundaries regions $\left(\partial \Gamma \equiv \eta=\bigcup_{\alpha=1}^{n} \eta_{\alpha}\right)$, where $\eta_{\alpha}=\partial \Gamma_{\alpha}$.

Point $Q$ is called a branch point of the manifold $\Gamma$, if it is a boundary point of at least two different regions $\Gamma_{\alpha}, \Gamma_{\beta}$ where $\alpha \neq \beta$.

Assumed that on $\Gamma$ given Borel measure, we determine the requirement that its restriction to each regions $\Gamma_{\alpha}$ coincides with the standard Lebesgue measure space $R^{d_{\alpha}}$. Then the space of square-integrable in the Lebesgue measure on the set of complex-valued functions $\Gamma$ admits the representation $L_{2}(\Gamma)=\oplus L_{2}\left(\Gamma_{\alpha}\right)$.

Let $C_{0,0}^{\infty}(\Gamma)$-vector space of infinitely differentiable complex-valued functions on $\Gamma$ with compact support not containing branch points of the manifold, and $\mathbf{L}_{0}=\oplus \mathbf{L}_{0}^{\alpha}$-linear operator defined on $C_{0}^{\infty}(\Gamma)$, by relation $\mathbf{L}_{0} u=\oplus\left\{\mathbf{L}_{0}^{\alpha} u_{\alpha}\right\}$, where

$$
\mathbf{L}_{0} \mathrm{u}=\frac{1}{\mathrm{~m}} \Delta \mathrm{u}+\mathrm{i}(\overrightarrow{\mathrm{B}}(\mathrm{x}), \nabla \mathrm{u})+\operatorname{idiv}(\overrightarrow{\mathrm{B}}(\mathrm{x}) \mathrm{u})+\mathrm{C}(\mathrm{x}) \mathrm{u},
$$

in which the functions $\mathrm{m}, \overrightarrow{\mathrm{B}}, \mathrm{C}$-real-valued, bounded and continuous everywhere except at the branching points of $\Gamma$, function $\mathrm{m}$ takes on each region $\Gamma_{\alpha}$ a constant value $\mathrm{m}_{\alpha}$, and $\mathrm{m}_{\alpha} \geq \mathrm{m}_{0}>0$, for all $\alpha=1, \cdots, \mathrm{n} ; \quad u \in C_{0}^{\infty}(\Gamma)$. Here $\left\{u_{\alpha}, \alpha=1, \cdots, n\right\}$-restricting a function $u$ on the region $\Gamma_{\alpha}$.

Definition: The linear self-adjoint operator $\mathbf{L}$ in the space $H=L_{2}(\Gamma)$ is called Hamiltonian of quantum system with mass $m$ in the electromagnetic filed $\{C, \vec{B}\}$ if $\mathbf{L}$ is self-adjoint extension of the operator $\mathbf{L}_{0}$.

We investigate the properties of the Cauchy problem for the Schrödinger equation

$$
i \frac{\partial \mathrm{u}(\mathrm{x}, \mathrm{t})}{\partial \mathrm{x}}=\mathbf{L u}(\mathrm{x}, \mathrm{t}) \text {, }
$$

with the initial condition

$$
\mathrm{u}(\mathrm{x},+0)=\mathrm{u}_{0}(\mathrm{x}), \mathrm{x} \in \Gamma \text {. }
$$

Here $\mathbf{L}$-symmetric operator in a Hilbert space $H=L_{2}(\Gamma)$, is an extension operator of $\mathbf{L}_{0}$, given on linear manifold $\mathrm{D}\left(\mathbf{L}_{0}\right)$ by the equation (2.1) or (2.2). The purpose of the article is to describe the set of all self-ad- 
joint extensions of the operator $\mathbf{L}_{0}$, that may act as generators of the unitary group of the Cauchy problem (2.3), (2.4) for the Schrödinger equation.

\section{Graph with One Vertex}

Graph $\Gamma$ with one vertex, is defined as the union of $n$ instances of semidirect $\Gamma_{j}=[0,+\infty), \quad j=1, \cdots, n$, with a common origin $Q$, called the vertex of the graph. Assumed that on $\Gamma$ given Borel measure defined by the requirement that its restriction to each semidirect $\Gamma_{j}$ coincides with the standard Lebesgue measure, then $L_{2}(\Gamma)=\oplus L_{2}\left(\Gamma_{j}\right)$. Let $C_{0,0}^{\infty}(\Gamma)$-vector space of infinitely differentiable complex-valued functions on $\Gamma$ with compact support not containing the point $Q$, and $\mathbf{L}_{0}=\oplus \mathbf{L}_{0}^{j}$-linear operator, defined on $C_{0}^{\infty}(\Gamma)$, by the relation $\mathbf{L}_{0} u=\oplus\left\{\mathbf{L}_{0}^{j} u_{j}\right\}$,

$$
\mathbf{L}_{0}^{j} u_{j}=\frac{1}{\mathrm{~m}_{j}} \Delta_{j} u_{j}+\mathrm{iB}_{\mathrm{j}}(\mathrm{x}) \frac{\partial u_{j}}{\partial \mathrm{x}}+\mathrm{i} \frac{\partial\left(\mathrm{B}_{j}(\mathrm{x}) u_{j}\right)}{\partial \mathrm{x}}+\mathrm{C}_{j}(\mathrm{x}) u_{j} .
$$

Here $\left\{u_{j}, \mathrm{j}=1, \cdots, n\right\}$-restriction of a function $u$ on semidirect $\Gamma_{\mathrm{j}}$.

Assumed that for all $j$ number $\mathrm{m}_{j}>0$ and the function $\mathrm{B}_{j}(\mathrm{x}), \mathrm{C}_{j}(\mathrm{x}) \in C^{1}\left(\Gamma_{j}, R\right)$ and $\mathrm{b}_{j}=\mathrm{B}_{j}(0)$ we denote in the point $Q$.

Operator $\mathbf{L}_{0}$ with domain of definition $\mathrm{D}\left(\mathrm{L}_{0}\right)=\mathrm{C}_{0,0}^{\infty}(\Gamma) \subset \mathrm{L}_{2}(\Gamma)$ is densely defined and symmetric. The domain $\mathrm{D}\left(\mathbf{L}_{0}^{*}\right)$ adjoint operator $\mathbf{L}_{0}^{*}$ is a linear subspace $\mathrm{D}\left(\mathbf{L}_{0}^{*}\right)=\oplus_{j=1}^{n} W_{2}^{2}\left(\Gamma_{j}\right):=W_{2}^{2}(\Gamma) \subset H$. The restriction of any function $u \in W_{2}^{2}(\Gamma)$ on semidirect $\Gamma_{j}, \quad j=1, \cdots, n$ possess boundary values at the vertex, which we denote by $u_{\mathrm{j}}(0)$, where the symbol $u(0)$ means $u(0)=\left(\begin{array}{llll}u_{1}(0) & u_{2}(0) & \cdots & u_{n}(0)\end{array}\right)^{\mathrm{T}} \in \mathbb{C}^{n}$. This is also true for the first derivatives of these restrictions, which use similar notation.

Von Neumann theorem $([9,10])$ provides a description of a set of self-adjoint extensions of symmetric operators. We obtain an explicit description of a set of self-adjoint extensions of the operator $\mathbf{L}_{0}$ in terms of conditions on a linear subspace in the space of boundary values

$$
G=\mathrm{D}\left(\mathbf{L}_{0}^{*}\right) / \overline{\mathrm{D}\left(\mathbf{L}_{0}\right)}=\left\{\left(u(0), u^{\prime}(0)\right)\right\}=\mathbb{C}^{2 n} .
$$

Theorem 1. Let $\mathrm{m}=1, \mathrm{~B}(\mathrm{x})=0$ and $\mathrm{C}(\mathrm{x})=0$. The operator $\mathbf{L}$ with domain

$$
\mathrm{D}(\mathbf{L})=\left\{u \in W_{2}^{2}(\Gamma): u^{\prime}(0)=A u(0)\right\},
$$

self-adjoint if and only if the matrix $A$ satisfies the equality $A=A^{*}$.

Proof. If $u \in \mathrm{D}(\mathbf{L})$ and $v \in \mathrm{D}\left(\mathbf{L}_{0}^{*}\right)$, then we have the equality

$$
(\mathbf{L} u, v)_{H}-\left(u, \mathbf{L}_{0}^{*} v\right)_{H}=\left(u(0), v^{\prime}(0)\right)_{\mathbb{C}^{n}}-\left(u^{\prime}(0), v(0)\right)_{\mathbb{C}^{n}} .
$$

Hence $(\mathbf{L} u, v)_{H}-\left(u, \mathbf{L}_{0}^{*} v\right)_{H}=\left(u(0), v^{\prime}(0)-A^{*} v(0)\right)_{\mathbb{C}^{n}}$.

Traces $u(0)$ take arbitrary values, therefore the equality $v^{\prime}(0)=A^{*} v(0)$ is necessary and sufficient for inclusion $v \in \mathrm{D}\left(\mathbf{L}^{*}\right)$, which proves Theorem 1 .

Corollary 1. If $M$ and $\mathcal{B}$-diagonal matrices and the matrix elements are defined by the formula $\left(\frac{1}{\mathrm{~m}_{i}} \delta_{i j}\right)_{n \times n},\left(\frac{1}{\mathrm{~b}_{i}} \delta_{i j}\right)_{n \times n} i, j=1, \cdots, n$ respectively, and $\mathrm{C}=\left(\mathrm{c}_{i j}\right)$, where $\mathrm{c}_{i j} \in L_{\infty}(\Gamma)$, then the operator $\mathbf{L}$ with domain $\mathrm{D}(\mathbf{L})=\left\{u \in W_{2}^{2}(\Gamma): u^{\prime}(0)=A u(0)\right\}$, self-adjoint if and only if the matrices $A, \quad \mathbf{M}$ and $\mathcal{B}$ satisfy the equality $A=\mathrm{M}^{-1} A^{*} \mathrm{M}-2 \mathrm{iM}^{-1} \mathcal{B}$.

Proof. If $u \in \mathrm{D}(\mathbf{L})$ and $v \in \mathrm{D}\left(\mathbf{L}_{0}^{*}\right)$, then we have the equality

$$
(\mathbf{L} u, v)_{H}-\left(u, \mathbf{L}_{0}^{*} v\right)_{H}=\left(u(0), \mathrm{M}^{\prime}(0)\right)_{\mathbb{C}^{n}}-\left(\mathrm{M} u^{\prime}(0), \bar{v}(0)\right)_{\mathbb{C}^{n}}-(2 \mathrm{i} u(0), \mathcal{B} \bar{v}(0))_{\mathbb{C}^{n}} .
$$

Hence

$$
(\mathbf{L} u, v)_{H}-\left(u, \mathbf{L}_{0}^{*} v\right)_{H}=\left(u(0), \mathrm{M} v^{\prime}(0)-A^{*} \mathrm{M} v(0)+2 i \mathcal{B} v(0)\right)_{\mathbb{C}^{n}} .
$$

Traces $u(0)$ take arbitrary values, therefore the equality $v^{\prime}(0)=\left(\mathrm{M}^{-1} A^{*} \mathrm{M}-2 \mathrm{i} \mathrm{M}^{-1} \mathcal{B}\right) v(0)$ is necessary and sufficient for inclusion $v \in \mathrm{D}\left(\mathbf{L}^{*}\right)$, which proves the corollary 1 .

Theorem 1 gives a description of a wide class of self-adjoint extensions of the operator $\mathbf{L}_{0}$, but does not de- 
scribe the totality of self-adjoint extensions. This makes the next theorem.

Theorem 2. The operator $\mathbf{L}$ is self-adjoint if and only if its domain of definition $\mathrm{D}(\mathbf{L})$ consists of the functions in the space $W_{2}^{2}(\Gamma)$, boundary values satisfy the equality $Z u^{\prime}(0)+A u(0)=0$, where rank of the matrix $(\mathrm{Z} \mid \mathrm{A})$ equals $n$ and the matrix is $\mathrm{ZA}^{*}$ is self-adjoint: $\mathrm{ZA}^{*}=A \mathrm{Z}^{*}$.

Proof. Let $\mathrm{m}=1, \mathrm{~B}(\mathrm{x})=0$ and $\mathrm{C}(\mathrm{x})=0$. We denote by $\left\{\left(\begin{array}{c}u(0) \\ u^{\prime}(0)\end{array}\right)_{2 n \times 1}=\boldsymbol{\Phi}_{2 n \times n} h_{n \times 1}\right\}$ set of solutions of linear equations

$$
\mathrm{Z} u^{\prime}(0)+A u(0)=0,
$$

where $\Phi_{2 n \times n}$ is the fundamental matrix and $h_{n \times 1}$ is a matrix of independent constants. Substituting each of the solutions of the fundamental equation $\mathrm{Zu}^{\prime}(0)+A u(0)=0$, specifying the domain, we obtain by following the relation of the fundamental matrix, with the matrix of the system of Equation (3.1)

$$
\boldsymbol{\Phi}^{\mathrm{T}}\left(\begin{array}{c}
A^{\mathrm{T}} \\
Z^{\mathrm{T}}
\end{array}\right)_{2 n \times n}=0_{n \times n}
$$

If $u \in \mathrm{D}(\mathbf{L})$ and domain of the operator $\mathbf{L}$ defined by a system of Equation (3.1), then for any $v \in \mathrm{D}\left(\mathbf{L}_{0}^{*}\right)$ rightly the equality

$$
(\mathbf{L} u, v)_{H}-\left(u, \mathbf{L}_{0}^{*} v\right)_{H}=\left\langle h, \Phi^{\mathrm{T}}\left(\begin{array}{cc}
0 & 1 \\
-1 & 0
\end{array}\right)_{2 n \times 2 n}\left(\begin{array}{c}
\bar{v}(0) \\
\overline{v^{\prime}}(0)
\end{array}\right)_{2 n \times 1}\right\rangle .
$$

Element $v \in \mathrm{D}\left(\mathbf{L}^{*}\right)$ satisfies condition

$$
(\mathbf{L} u, v)_{H}-\left(u, \mathbf{L}_{0}^{*} v\right)_{H}=0 .
$$

Let $\overline{\mathrm{V}}=\left(\begin{array}{lll}\bar{v}_{1} & \cdots & \bar{v}_{n} \\ \bar{v}_{1}^{\prime} & \cdots & \bar{v}_{n}^{\prime}\end{array}\right)_{2 n \times n}$-basis in the linear $\mathrm{D}\left(\mathbf{L}^{*}\right) / \overline{\mathrm{D}\left(\mathbf{L}_{0}\right)}$, then each column of matrix $\overline{\mathrm{V}}$ satisfies (3.3), and therefore

$$
\boldsymbol{\Phi}^{\mathrm{T}}\left(\begin{array}{cc}
0 & 1 \\
-1 & 0
\end{array}\right)_{2 n \times 2 n} \overline{\mathrm{V}}_{2 n \times n}=0_{n \times n}
$$

Of (3.2) and (3.4), it follows that the matrix $\mathrm{V}$ can be selected $\mathrm{V}=\left(\begin{array}{c}-\mathrm{Z}^{*} \\ A^{*}\end{array}\right)$.

The operator $\mathbf{L}$ is self-adjoint if and only if $\mathrm{D}(\mathbf{L})=\mathrm{D}\left(\mathbf{L}^{*}\right)$, so if $\mathrm{V}$-matrix of the columns of the basis vectors in the subspace $\mathrm{D}\left(\mathbf{L}^{*}\right) / \overline{\mathrm{D}\left(\mathbf{L}_{0}\right)}$, then $\mathrm{D}(\mathbf{L})=\mathrm{D}\left(\mathbf{L}^{*}\right)$ if and only if $\mathrm{V}$ is also the matrix of the columns of the basis vectors in the subspace $\mathrm{D}(\mathbf{L}) / \overline{\mathrm{D}\left(\mathbf{L}_{0}\right)}$, that is, any of its column satisfies the system of Equaation (3.1). And this is equivalent to the system of equations $\left(\begin{array}{ll}A & Z\end{array}\right)\left(\begin{array}{c}-Z^{*} \\ A^{*}\end{array}\right)=0$, which proves Theorem 2 .

Theorem 3. The operator $\mathbf{L}$ is self-adjoint if and only if its domain of definition $\mathrm{D}(\mathbf{L})$ consists of the functions in the space $W_{2}^{2}(\Gamma)$, boundary values satisfy the equality $A_{1} u^{\prime}(0)+A_{0} u(0)=0$, where rank of the matrix $\left(A_{1} \mid A_{0}\right)$ equals $n$ and the matrix is $A_{0} A_{1}^{*}$ is self-adjoint: $A_{0} \mathrm{M}^{-1} A_{1}^{*}=A_{1} \mathrm{M}^{-1}\left(A_{0}^{*}+2 i \mathcal{B} \mathrm{M}^{-1} A_{1}^{*}\right)$.

Proof. Let $\mathrm{m} \neq 0, \mathrm{~B}(\mathrm{x}) \neq 0$ and $\mathrm{C}(\mathrm{x}) \neq 0$. We denote by $\left\{\left(\begin{array}{c}u(0) \\ u^{\prime}(0)\end{array}\right)_{2 n \times 1}=\boldsymbol{\Phi}_{2 n \times n} h_{n \times 1}\right\}$ set of solutions of linear equations

$$
A_{1} u^{\prime}(0)+A_{0} u(0)=0
$$

where $\boldsymbol{\Phi}_{2 n \times n}$ is the fundamental matrix and $h_{n \times 1}$ is a matrix of independent constants. Substituting each of the solutions of the fundamental equation $A_{1} u^{\prime}(0)+A_{0} u(0)=0$, specifying the domain, we obtain by following the 
relation of the fundamental matrix, with the matrix of the system of Equation (3.5)

$$
\Phi^{\mathrm{T}}\left(\begin{array}{l}
A_{0}^{\mathrm{T}} \\
A_{1}^{\mathrm{T}}
\end{array}\right)_{2 n \times n}=0_{n \times n} .
$$

If $u \in \mathrm{D}(\mathbf{L})$ and domain of the operator $\mathbf{L}$ defined by a system of Equation (3.5), then for any $v \in \mathrm{D}\left(\mathbf{L}_{0}^{*}\right)$ rightly the equality

$$
(\mathbf{L} u, v)_{H}-\left(u, \mathbf{L}_{0}^{*} v\right)_{H}=\left\langle h, \Phi^{\mathrm{T}}\left(\begin{array}{cc}
-2 \mathrm{i} \mathcal{B} & \mathbf{M} \\
-\mathrm{M} & 0
\end{array}\right)_{2 n \times 2 n}\left(\begin{array}{c}
\bar{v}(0) \\
\overline{v^{\prime}}(0)
\end{array}\right)_{2 n \times 1}\right\rangle .
$$

Element $v \in \mathrm{D}\left(\mathbf{L}^{*}\right)$ satisfies condition

$$
(\mathbf{L} u, v)_{H}-\left(u, \mathbf{L}_{0}^{*} v\right)_{H}=0
$$

Let $\overline{\mathrm{V}}=\left(\begin{array}{ccc}\bar{v}_{1} & \cdots & \bar{v}_{n} \\ \overline{v_{1}^{\prime}} & \cdots & \bar{v}_{n}^{\prime}\end{array}\right)_{2 n \times n}$-basis in the linear $\mathrm{D}\left(\mathbf{L}^{*}\right) / \overline{\mathrm{D}\left(\mathbf{L}_{0}\right)}$, then each column of matrix $\overline{\mathrm{V}}$ satisfies (3.7), and therefore

$$
\boldsymbol{\Phi}^{\mathrm{T}}\left(\begin{array}{cc}
-2 \mathrm{i} \mathcal{B} & \mathrm{M} \\
-\mathrm{M} & 0
\end{array}\right)_{2 n \times 2 n} \overline{\mathrm{V}}_{2 n \times n}=0_{n \times n}
$$

Of (3.6) and (3.8), it follows that the matrix $V$ can be selected $V=\left(\begin{array}{c}-\mathrm{M}^{-1} A_{1}^{*} \\ \mathrm{M}^{-1} A_{0}^{*}+2 i \mathrm{M}^{-1} \mathcal{B M}^{-1} A_{1}^{*}\end{array}\right)$.

The operator $\mathbf{L}$ is self-adjoint if and only if $\mathrm{D}(\mathbf{L})=\mathrm{D}\left(\mathbf{L}^{*}\right)$, so if $\mathrm{V}$-matrix of the columns of the basis vectors in the subspace $\mathrm{D}\left(\mathbf{L}^{*}\right) / \overline{\mathrm{D}\left(\mathbf{L}_{0}\right)}$, then $\mathrm{D}(\mathbf{L})=\mathrm{D}\left(\mathbf{L}^{*}\right)$ if and only if $\mathrm{V}$ is also the matrix of the columns of the basis vectors in the subspace $\mathrm{D}(\mathbf{L}) / \overline{\mathrm{D}\left(\mathbf{L}_{0}\right)}$, that is, any of its column satisfies the system of Equation (3.5). And this is equivalent to the system of equations

$$
\left(\begin{array}{ll}
A_{0} & A_{1}
\end{array}\right)\left(\begin{array}{c}
-\mathrm{M}^{-1} A_{1}^{*} \\
\mathrm{M}^{-1} A_{0}^{*}+2 i \mathrm{M}^{-1} \mathcal{B} \mathrm{M}^{-1} A_{1}^{*}
\end{array}\right)=0,
$$

which proves Theorem 3.

\section{Graph with Multiple Vertices}

In the present article, a graph with multiple vertices is understood by one-dimensional cellular of complex [3]. Let graph $\Gamma$, be a collection of $n$ vertices $Q_{1}, \cdots, Q_{n}$, from each of which proceeds $r_{j}, r_{j} \in N$ edges $\Gamma_{j}^{i}$, representing the infinity semidirect or line segments that connect vertex $Q_{j}$ with other vertices. We fix on each edges $\Gamma_{j}$ parametrization of the natural parameters. In this case, the edges of semidirect parameter increases from the boundary points and the edges of intervals, the orientation is chosen arbitrarily. Let $d_{j}$-initial point of the edges semidirect, $\mathrm{a}_{j}$-initial point of edges $\Gamma_{j}$ interval, $\mathrm{b}_{j}$-end point of edges $\Gamma_{j}$ interval. Let $\mathrm{c}_{k}, k=1, \cdots, \mathrm{N}$-the collection of all boundary points of the edges $\Gamma_{1}, \cdots, \Gamma_{n}$. We define the function $\mathrm{s}$ on the set $\left\{\mathrm{c}_{k}\right\}$ so that $s\left(\mathrm{c}_{k}\right)=1$ if $\mathrm{c}_{k}$-beginning of edges and $s\left(\mathrm{c}_{k}\right)=-1$ if $\mathrm{c}_{k}$-end of the edges, denoted by $S$ diagonal matrix with numbers $s\left(\mathrm{c}_{k}\right)$ on the diagonal.

We introduce the operators $\mathbf{L}_{0}, \mathbf{L}_{0}^{*}$ and the space $G$ of boundary values of functions from $\mathrm{D}\left(\mathbf{L}_{0}^{*}\right)$ and their derivatives, linearly isomorphic to space $\mathbb{C}^{2 N}$. Through $u\left(c_{j}\right)$ we denote the collection limit function values on edges of the boundary, which is the point $c_{j}$, and by $u(c)$ denote by $\mathrm{N}$-dimensional vector of $\left(u\left(c_{1}\right) \quad u\left(c_{2}\right) \cdots u\left(c_{\mathrm{N}}\right)\right)^{\mathrm{T}}$, for the vector limit values of the derivative $u^{\prime}(c)$ use similar notation, and let $b_{j}=\mathrm{B}(c)$ denoted in $Q$.

Theorem 4. Let $\mathrm{m}=1, \mathrm{~B}(\mathrm{x})=0$ and $\mathrm{C}(\mathrm{x})=0$. The operator $\mathbf{L}$ with domain

$$
\mathrm{D}(\mathbf{L})=\left\{u \in W_{2}^{2}(\Gamma): u^{\prime}(c)=A u(c)\right\},
$$

adjoint if and only if the matrix $A$ satisfies the equality $A=S A^{*} S$.

Proof. If $u \in \mathrm{D}(\mathbf{L})$ and $v \in \mathrm{D}\left(\mathbf{L}_{0}^{*}\right)$, then we have the equality 


$$
(\mathbf{L} u, v)_{H}-\left(u, \mathbf{L}_{0}^{*} v\right)_{H}=\left(S u(c), v^{\prime}(c)\right)_{\mathbb{C}^{2 n}}-\left(S u^{\prime}(c), v(c)\right)_{\mathbb{C}^{2 n}}
$$

Hence

$$
(\mathbf{L} u, v)_{H}-\left(u, \mathbf{L}_{0}^{*} v\right)_{H}=\left(u(c), S v^{\prime}(c)-A^{*} S v(c)\right)_{\mathbb{C}^{2 n}} .
$$

Traces $u(c)$ take arbitrary values, therefore the equality $v^{\prime}(c)=S A^{*} S v(c)$ is necessary and sufficient for inclusion $v \in \mathrm{D}\left(\mathbf{L}^{*}\right)$, which proves Theorem 4 .

Corollary 2. If $M$ and $\mathcal{B}$-diagonal matrices and the matrix elements are defined by the formula $\left(\frac{1}{\mathrm{~m}_{i}} \delta_{i j}\right)_{2 n \times 2 n},\left(\frac{1}{\mathrm{~b}_{i}} \delta_{i j}\right)_{2 n \times 2 n} i, j=1, \cdots, 2 n$ respectively, and $\mathrm{C}=\left(\mathrm{c}_{i j}\right)$, where $\mathrm{c}_{i j} \in L_{\infty}(\Gamma)$, then the operator $\mathbf{L}$ with domain $\mathrm{D}(\mathbf{L})=\left\{u \in W_{2}^{2}(\Gamma): u^{\prime}(c)=A u(c)\right\}$, self-adjoint if and only if the matrices $A, \quad \mathbf{M}$ and $\mathcal{B}$ satisfy the equality $A=\mathrm{M}^{-1} S A^{*} S M-2 \mathrm{iM}^{-1} S \mathcal{B} S$.

Proof. If $u \in \mathrm{D}(\mathbf{L})$ and $v \in \mathrm{D}\left(\mathbf{L}_{0}^{*}\right)$, then we have the equality

$$
(\mathbf{L} u, v)_{H}-\left(u, \mathbf{L}_{0}^{*} v\right)_{H}=\left(S(u(c))^{\mathrm{T}}, \mathbf{M} \overline{v^{\prime}}(c)\right)-\left(S\left(u^{\prime}(c)\right)^{\mathrm{T}}, \mathbf{M} \bar{v}(c)\right)-\left(2 \mathrm{i} S(u(c))^{\mathrm{T}}, \boldsymbol{B} \bar{v}(c)\right) .
$$

Hence

$$
(\mathbf{L} u, v)_{H}-\left(u, \mathbf{L}_{0}^{*} v\right)_{H}=\left((u(c))^{\mathrm{T}}, S \mathrm{M} v^{\prime}(c)-A^{*} \mathrm{~S} \operatorname{Mv}(c)+2 \mathrm{i} \mathcal{B S} v(c)\right) .
$$

Traces $(u(c))^{\mathrm{T}}$ take arbitrary values, therefore the equality $v^{\prime}(\mathrm{c})=\left(\mathrm{M}^{-1} S A^{*} S \mathrm{M}-2 \mathrm{iM}{ }^{-1} S \mathcal{B} S\right) v(c)$ is necessary and sufficient for inclusion $v \in \mathrm{D}\left(\mathbf{L}^{*}\right)$, which proves the corollary 2 .

\section{Graph with One Vertex and with a Countable Set of Rays}

Description of this graph is defined by the following structures [11]. In this case we denote by $\mu$-locally finite non-negative countably additive measure on $N$ such that $\mu(k)=\mu_{k}>0$, denoted by $L_{2}\left(N, 2^{N}, \mu\right.$, $\left.\mathbb{C}\right)-$ Hilbert space of boundary values with the norm

$$
\left\|\left\{u_{n}\right\}\right\|_{L_{2, \mu}}^{2}=\int_{N}\left|u_{n}\right|^{2} \mathrm{~d} \mu(n)=\sum_{k=1}^{\infty}\left|u_{k}\right|^{2} \mu(k) .
$$

The restriction of any function on semidirect possesses the boundary values at the vertex:

$u(0)=\left(\begin{array}{lll}u_{1}(0) \quad \cdots & u_{n}(0) \quad \cdots\end{array}\right)^{\mathrm{T}} \in L_{2, \mu}$. This is also true for the first derivatives of these restrictions $u^{\prime}(0)$. We denote by $\Lambda, \mathbf{M}$ and $\mathcal{B}$ diagonal matrices and their matrix elements are given by the formula $\left(\mu_{i} \delta_{i j}\right)$, $\left(\frac{1}{\mathrm{~m}_{i}} \delta_{i j}\right)$ and $\left(b_{i} \delta_{i j}\right)$ respectively.

Theorem 5. Let $\mu_{k}=1, \quad \mathrm{~m}=1, \mathrm{~B}(\mathrm{x})=0$ and $\mathrm{C}(\mathrm{x})=0$. The operator $\mathbf{L}$ with domain $\mathrm{D}(\mathbf{L})=\left\{u \in W_{2}^{2}(\Gamma): u^{\prime}(0)=A u(0)\right\}$, self-adjoint if and only if the operator $A$ is self-adjoint in the space $l_{2}$.

Proof. If $u \in \mathrm{D}(\mathbf{L})$ and $v \in \mathrm{D}\left(\mathbf{L}_{0}^{*}\right)$, then we have the equality

$$
(\mathbf{L} u, v)_{H}-\left(u, \mathbf{L}_{0}^{*} v\right)_{H}=\left((u(0))^{\mathrm{T}}, \Lambda \overline{v^{\prime}}(0)\right)-\left(\left(u^{\prime}(0)\right)^{\mathrm{T}}, \Lambda \bar{v}(0)\right)=\left((\mathrm{u}(0))^{\mathrm{T}}, \Lambda \overline{v^{\prime}}(0)\right)-\left((\mathrm{u}(0))^{\mathrm{T}}, \mathrm{A}^{\mathrm{T}} \Lambda \bar{v}(0)\right) .
$$

Hence $(\mathbf{L} u, v)_{H}-\left(u, \mathbf{L}_{0}^{*} v\right)_{H}=\left((u(0))^{\mathrm{T}}, \Lambda \overline{v^{\prime}}(0)-A^{\mathrm{T}} \Lambda \bar{v}(0)\right)$.

Traces $(u(0))^{\mathrm{T}}$ take arbitrary values in the space $L_{2}\left(N, 2^{N}, \mu, \mathbb{C}\right)$, therefore the equality $v^{\prime}(0)=\Lambda^{-1} A^{*} \Lambda v(0)$ is necessary and sufficient for inclusion $v \in \mathrm{D}\left(\mathbf{L}^{*}\right)$, which proves Theorem 5 .

Corollary 3. If $\mu_{k} \in l_{1}, 1 / \mathrm{m}_{k}, \mathrm{~m}_{k}, b_{k} \in l_{\infty}$ and $\Lambda, \mathcal{B}$ and $\mathrm{M}$ are operators in the space $L_{2}\left(N, 2^{N}, \mu, \mathbb{C}\right)$, given by diagonal matrices with elements $\mu_{k}, b_{k}, 1 / \mathrm{m}_{k}$ on the diagonal, respectively, $\mathrm{C}=\left(\mathrm{c}_{i j}\right)$, where $\mathrm{c}_{i j} \in L_{\infty}(\Gamma)$. Then the operator $\mathbf{L}$ with domain $\mathrm{D}(\mathbf{L})=\left\{u \in W_{2}^{2}(\Gamma): u^{\prime}(0)=A u(0)\right\}$, self-adjoint if and only if the operators $A, \quad \mathrm{M}$ and $\mathcal{B}$ acting in the space $l_{2}$, satisfy the equality

$$
\Lambda \mathrm{MA}=A^{*} \Lambda \mathrm{M}-2 \mathrm{i} \Lambda \mathcal{B}
$$


Proof. If $u \in \mathrm{D}(\mathbf{L})$ and $v \in \mathrm{D}\left(\mathbf{L}_{0}^{*}\right)$, then we have the equality

$$
(\mathbf{L} u, v)_{H}-\left(u, \mathbf{L}_{0}^{*} v\right)_{H}=\left((u(0))^{\mathrm{T}}, \Lambda \mathrm{M} \overline{v^{\prime}}(0)\right)-\left(\left(u^{\prime}(0)\right)^{\mathrm{T}}, \Lambda \mathrm{M} \bar{v}(0)\right)-\left(2 \mathrm{i}(u(0))^{\mathrm{T}}, \Lambda \mathcal{B} \bar{v}(0)\right) .
$$

Hence $(\mathbf{L} u, v)_{H}-\left(u, \mathbf{L}_{0}^{*} v\right)_{H}=\left[\Lambda \mathrm{M} \overline{v^{\prime}}(0)-A^{\mathrm{T}} \Lambda \mathrm{M} \bar{v}(0)-2 \mathrm{i} \Lambda \mathcal{B} \bar{v}(0)\right](u(0))^{\mathrm{T}}$.

Traces $(u(0))^{\mathrm{T}}$ take arbitrary values in the space $L_{2}\left(N, 2^{N}, \mu, \mathbb{C}\right)$, therefore the equality

$\Lambda \mathrm{M} v^{\prime}(0)=\left(A^{*} \Lambda \mathrm{M}-2 \mathrm{i} \Lambda \mathcal{B}\right) v(0)$ is necessary and sufficient for inclusion $v \in \mathrm{D}\left(\mathbf{L}^{*}\right)$, as $\mathbf{L}=\mathbf{L}^{*}$ if and only if $\mathrm{D}(\mathbf{L})=\mathrm{D}\left(\mathbf{L}^{*}\right)$, then for the self-adjoint operator $\mathbf{L}$ is necessary and sufficient to satisfy the Equality (5.1).

\section{Schrödinger Operators on Branched Manifolds}

The assumption $\mathbf{H}$. Let the function $\mathrm{m}$ takes the constant values $\mathrm{m}_{\alpha}>0$ on each region $\Gamma_{\alpha}$ for all $\alpha \in\{1, \cdots, n\}$, and satisfy condition $\overrightarrow{\mathrm{B}_{\alpha}}(x) \in C^{1}\left(\Gamma_{\alpha}, R^{d_{\alpha}}\right) \cap C\left(\bar{\Gamma}_{\alpha}, R^{d_{\alpha}}\right), \quad \mathrm{C}_{\alpha}(x) \in C\left(\bar{\Gamma}_{\alpha}, R\right)$. Through $\overrightarrow{\mathrm{b}_{\alpha}}=\left.\overrightarrow{\mathrm{B}_{\alpha}}\right|_{\eta_{\alpha}}$ we denote the limiting values of the vector function $\overrightarrow{\mathrm{B}_{\alpha}}$ on the boundary $\eta_{\alpha}$.

The operator $\mathbf{L}_{0}$ with domain $\mathrm{D}\left(\mathrm{L}_{0}\right)=\mathrm{C}_{0,0}^{\infty}(\Gamma) \subset L_{2}(\Gamma)$, densely defined and symmetric. The domain $\mathrm{D}\left(\mathbf{L}_{0}^{*}\right)$ adjoint operator $\mathbf{L}_{0}^{*}$ is a linear subspace $\mathrm{D}\left(\mathbf{L}_{0}^{*}\right)=\oplus_{\alpha=1}^{n} W_{2}^{2}\left(\Gamma_{\alpha}\right):=W_{2}^{2}(\Gamma) \subset H$.

Let the components $\Gamma_{\alpha}$ manifold $\Gamma$ constitute a $m$ semidirect, $k$ finite intervals and $\mathrm{N}-(m+k)$ regions. In the case of one-dimensional region $\Gamma_{\alpha}$ boundary value $\left.u_{\alpha}\right|_{\eta_{\alpha}}$ is a set of complex numbers on the boundary $\eta_{\alpha}$, represents one or two points. In the case $d_{\alpha} \geq 2$ boundary value $\left.u_{\alpha}\right|_{\eta_{\alpha}}$ is an element of the space $W_{2}^{3 / 2}\left(\eta_{\alpha}\right)$. According to the trace theorem $\left.u\right|_{\eta} \in W_{2}^{3 / 2}(\eta)=\oplus_{\alpha=1}^{N} W_{2}^{3 / 2}\left(\eta_{\alpha}\right)$ ([12]). Through $\left.u\right|_{\eta}$ denote the collection of $\left(\left.u\right|_{\eta_{1}}, \cdots,\left.u\right|_{\eta_{N}}\right)^{\mathrm{T}}$ limit values function $u$ on the boundary $\eta$. Similarly, the limit value of the derivative $\frac{\partial u_{\alpha}}{\partial n_{\alpha}}$ constriction $u_{\alpha}$ in the direction of the outer normal $n_{\alpha}$ to boundary $\eta_{\alpha}$ in the case semidirect $\eta_{\alpha}$ represents a an element of space $\mathbb{C}$, in case of a limited interval-element of space $\mathbb{C}^{2}$, and in the case of dimension $d_{\alpha} \geq 2$-element of space $W_{2}^{1 / 2}\left(\eta_{\alpha}\right)$.

The boundary values of the normal derivative is denoted by $\left.\frac{\partial u}{\partial n}\right|_{\eta} \equiv\left(\left.\frac{\partial u}{\partial n_{1}}\right|_{\eta_{1}}, \cdots,\left.\frac{\partial u}{\partial n_{N}}\right|_{\eta_{N}}\right)^{\mathrm{T}}$, where $n_{\alpha}$ is vector of the external relative to $\Gamma_{\alpha}$ normal to the $\eta_{\alpha}, \quad \alpha=1, \cdots, N$.

We introduce the Hilbert space

$$
h=L_{2}(\eta)=\oplus_{\alpha=1}^{N} L_{2}\left(\eta_{\alpha}\right)=\mathbb{C}^{m+2 k} \oplus_{\alpha=m+k+1}^{N} L_{2}\left(\eta_{\alpha}\right) .
$$

We define space of boundary values $G=h^{3 / 2} \oplus h^{1 / 2}$, where $h^{3 / 2}=\mathbb{C}^{m+2 k} \oplus_{\alpha=m+k+1}^{N} W_{2}^{3 / 2}\left(\eta_{\alpha}\right)$ and similarly,

$h^{1 / 2}=\mathbb{C}^{m+2 k} \oplus_{\alpha=m+k+1}^{N} W_{2}^{1 / 2}\left(\eta_{\alpha}\right)$.
Boundary value $\left.u\right|_{\eta}$ function $u \in W_{2}^{2}(\Gamma)$ is an element of the space $h^{3 / 2}$, and the boundary value $\left.\frac{\partial u}{\partial n}\right|_{\eta}$
its normal derivative-an element of the space $h^{1 / 2}$. its normal derivative-an element of the space $h^{1 / 2}$.

We introduce in the space $h$ operators $M$ and $F$. Operator $M$ acts on each element $v \in h$ as an operator of multiplication by a function

$$
\mu(\xi)=\left\{\begin{array}{l}
\frac{1}{\mathrm{~m}_{\alpha}}, \xi \in \eta_{\alpha}, \\
\alpha \in\{1, \cdots, n\} .
\end{array}\right.
$$

And operator $F$ acts on each element $v \in h$ as an operator of multiplication by a function

$$
\beta(\xi)=\left\{\begin{array}{l}
\left(\overrightarrow{b_{\alpha}}(\xi), \overrightarrow{n_{\alpha}}(\xi)\right), \xi \in \eta_{\alpha}, \\
\alpha \in\{1, \cdots, n\}
\end{array}\right.
$$


Theorem 6. Let performed assumption $\mathbf{H}$ about functions $\mathrm{m}, \overrightarrow{\mathrm{B}}, \mathrm{C}$ and $\mathrm{m}=1, \overrightarrow{\mathrm{b}_{\alpha}}=0$ for any $\alpha \in\{1, \cdots, n\}$. Let A-linear operator in space $h$ with a dense domain $h^{3 / 2}$, the values of which belongs in linear manifold $h^{1 / 2}$. Let $\mathrm{D}_{\mathrm{A}}$-linear manifold of functions $u \in W_{2}^{2}(\Gamma)$, boundary values are related to the boundary values of the derivatives in the direction of the outward normal by relation

$$
\left.\frac{\partial u}{\partial n}\right|_{\eta}=\left.\mathrm{A} u\right|_{\eta} .
$$

Then the self-adjoint operator $\mathbf{L}_{\mathrm{A}}=\left.\left(\mathbf{L}_{0}^{*}\right)\right|_{\mathrm{D}_{\mathrm{A}}}$ is necessary and sufficient to satisfy the equality $A=\mathrm{A}^{*}$.

Proof. Since $\mathrm{m}=1, \overrightarrow{\mathrm{b}_{\alpha}}=0$ for any $\alpha$, then from conditions $u \in \mathrm{D}\left(\mathbf{L}_{\mathrm{A}}\right)$ and $v \in \mathrm{D}\left(\mathbf{L}_{0}^{*}\right)$ then we have the equality

$$
\left(\mathbf{L}_{\mathrm{A}} u, v\right)_{h}-\left(u, \mathbf{L}_{0}^{*} v\right)_{h}=\left(\left.\frac{\partial u}{\partial n}\right|_{\eta},\left.v\right|_{\eta}\right)_{h}-\left(\left.u\right|_{\eta},\left.\frac{\partial v}{\partial n}\right|_{\eta}\right)_{h}
$$

Hence

$$
\left(\mathbf{L}_{\mathrm{A}} u, v\right)_{h}-\left(u, \mathbf{L}_{0}^{*} v\right)_{h}=\left(\left.u\right|_{\eta},\left.\mathrm{A}^{*} v\right|_{\eta}-\left.\frac{\partial v}{\partial n}\right|_{\eta}\right)_{h}
$$

Traces $\left.u\right|_{\eta}$ take arbitrary values, therefore the equality $\left.\frac{\partial v}{\partial n}\right|_{\eta}=\left.\mathrm{A}^{*} v\right|_{\eta}$ is necessary and sufficient for inclusion $v \in \mathrm{D}\left(\mathbf{L}_{\mathrm{A}}^{*}\right)$. Since the domain of definition operator $\mathbf{L}_{\mathrm{A}}$ is determined by the equation $\left.\frac{\partial u}{\partial n}\right|_{\eta}=\left.\mathrm{A} u\right|_{\eta}$, that $\mathbf{L}_{\mathrm{A}}=\mathbf{L}_{\mathrm{A}}^{*}$ then implies that $\mathrm{A}=\mathrm{A}^{*}$.

Corollary 4. Let performed assumption $\mathbf{H}$ about functions $\mathrm{m}, \overrightarrow{\mathrm{B}}$, C. Let A-linear operator in space $h$ with a dense domain $h^{3 / 2}$, the values of which belongs in linear manifold $h^{1 / 2}$. Let $D_{\mathrm{A}}$-linear manifold of functions $u \in W_{2}^{2}(\Gamma)$, boundary values are related to the boundary values of the derivatives in the direction of the outward normal by relation

$$
\left.\frac{\partial u}{\partial n}\right|_{\eta}=\left.\mathrm{A} u\right|_{\eta}
$$

Then the self-adjoint operator $\mathbf{L}_{\mathrm{A}}=\left.\left(\mathbf{L}_{0}^{*}\right)\right|_{\mathrm{D}_{\mathrm{A}}}$ is necessary and sufficient to satisfy the equality $M A=\mathrm{A}^{*} M-2 \mathrm{i} F$.

Proof. Since performed assumption $\mathbf{H}$, then from conditions $u \in \mathrm{D}\left(\mathbf{L}_{\mathrm{A}}\right)$ and $v \in \mathrm{D}\left(\mathbf{L}_{0}^{*}\right)$ then we have the equality

$$
\left(\mathbf{L}_{\mathrm{A}} u, v\right)_{h}-\left(u, \mathbf{L}_{0}^{*} v\right)_{h}=\left(\left.\frac{\partial u}{\partial \eta}\right|_{\eta},\left.M v\right|_{\eta}\right)_{h}-\left(\left.u\right|_{\eta},\left.M \frac{\partial v}{\partial n}\right|_{\eta}\right)_{h}+2 \mathrm{i}\left(\left.u\right|_{\eta},\left.F v\right|_{\eta}\right)_{h} .
$$

Hence

$$
\left(\mathbf{L}_{\mathrm{A}} u, v\right)_{h}-\left(u, \mathbf{L}_{0}^{*} v\right)_{h}=\left(\left.u\right|_{\eta},\left.\mathrm{A}^{*} M v\right|_{\eta}-\left.M \frac{\partial v}{\partial n}\right|_{\eta}-\left.2 \mathrm{i} F v\right|_{\eta}\right)_{h} .
$$

Traces $\left.u\right|_{\eta}$ take arbitrary values, therefore the equality $\left.M \frac{\partial v}{\partial n}\right|_{\eta}=\left.\left(\mathrm{A}^{*} M-2 \mathrm{i} F\right) v\right|_{\eta}$ is necessary and sufficient for inclusion $v \in \mathrm{D}\left(\mathbf{L}_{\mathrm{A}}^{*}\right)$. Since the domain of definition operator $\mathbf{L}_{\mathrm{A}}$ is determined by the equation $\left.\frac{\partial u}{\partial n}\right|_{\eta}=\left.\mathrm{A} u\right|_{\eta}$,

that $\mathbf{L}_{\mathrm{A}}=\mathbf{L}_{\mathrm{A}}^{*}$ then implies that $M \mathrm{~A}=\mathrm{A}^{*} M-2 \mathrm{i} F$. 


\section{Conclusion}

In this paper we describe the set of all Schrödinger operators on graph and branched manifold, defined as a self-adjoint extension of the operator, originally defined on smooth functions with supports, not contained in the branch points manifold. Thus, given a description of the various options, we determine the Laplace operator on the space of the functions defined on a branched manifold. Description of the definition of each of the self-adjoint extensions is given in terms of linear relations satisfied by the limit at the branch points and the boundary points of the graph function value in the domain of operator and the its derivative. Each of the Laplace operators corresponds to the Markov process, whose behavior in a neighborhood of branch points, we determined by the choice of the domain of the Laplace operator, obtained in this paper results, which is an extension of the study work [8] describes the self-adjoint extensions of a graph with a single vertex and two edges, to the case of a graph with an arbitrary number of edges. In addition, this paper summarizes the results of [6] in the case of Laplace operators, for which the linear relation in the space of boundary values that define the domain of the operator, do not admit the possibility of expressing the limit function values at the boundary points and branch points of the graph of the limiting values of its derivative.

\section{REFERENCES}

[1] Y. V. Pokorny, O. M. Penkin, V. L. Pryadier, A. V. Borovskikh, K. P. Lazarev and S. A. Shabrov, "Differential Equations on Geometric Graphs,” M. FIZMATLIT, 2004.

[2] V. L. Chernyshev and A. I. Shafarevich, "Semiclassical Spectrum of the Schrödinger Operator on a Geometric Graph,” Mathematical Notes, Vol. 82, No. 3-4, 2007, pp. 542-554. http://dx.doi.org/10.1134/S0001434607090313

[3] O. M. Penkin and Y. V. Pokornyi, “On Some Qualitative Properties of Equations for the One-Dimensional CW-complex," Mathematical Notes, Vol.59, No. 5, 1996, pp. 562-565. http://dx.doi.org/10.1007/BF02308827

[4] A. A. Tolchennikov, V. L. Chernyshev and A. I. Shafarevich, "Asymptotic Properties and Classical Dynamical Systems in Quantum Problems on Singular Spaces,” Nelineinaya Dinamika, Vol. 6, No. 3, 2010, pp. 623-638.

[5] G. G. Amosov and V. Z. Sakbaev, "On Self-Adjoint Extensions of Schrödinger Operators Degenerating on a Pair of Half-Lines and the Corresponding Markovian Cocycles,” Mathematical Notes, Vol. 76, No. 3, 2004, pp. 315-322. http://dx.doi.org/10.1023/B:MATN.0000043458.91218.7b

[6] V. Z. Sakbaev and O. G. Smolyanov, "Dynamics of a Quantum Particle with Discontinuous Position-Dependent Mass,” Doklady Mathematics, Vol. 82, No. 1, 2010, pp. 630-633. http://dx.doi.org/10.1134/S1064562410040332

[7] V. Z. Sakbaev and O. G. Smolyanov, "Diffusion and Quantum Dynamics on Graphs,” Doklady Mathematics, Vol. 88, No. 1, 2013, pp. 404-408. http://dx.doi.org/10.1134/S1064562413040108

[8] M. Gadella, S. Kuru and J. Negro, “Self-Adjoint Hamiltonians with a Mass Jump: General Matching Conditions,” Physics Letters A, 2007, Vol. 362, No. 4, pp. 265-268. http://dx.doi.org/10.1016/j.physleta.2006.10.029

[9] M. Reed and B. Simon, "Methods of Modern Mathematical Physics '1. Functional Analysis',” Academic Press, New York London, 1972.

[10] T. Kato, “Perturbation Theory for Linear Operators,” Springer-Verlag, Berlin, Heidelberg, New York, 1966.

[11] N. Dunford and J. T. Schwartz, “Linear Operators ‘Part I: General Theory’”” Interscience Publishers, New York, London, 1958.

[12] G. N. Jakovlev, “Traces of Functions in the Space $W_{p}^{l}$ on Piecewise Smooth Surfaces," Mathematics of the USSR-Sbornik, Vol. 3, No. 4, 1967, pp. 481-497. http://dx.doi.org/10.1070/SM1967v003n04ABEH002758 